\title{
Ruptured Internal Carotid Artery Aneurysm Presenting with Catastrophic Epistaxis after Repeated Stereotactic Radiotherapies for Anterior Skull Base Tumor: Case Reports and Review of the Literature
}

\author{
Koji Fujita ${ }^{1}$ Manabu Tamura ${ }^{2}$ Osamu Masuo ${ }^{1}$ Takahiro Sasaki ${ }^{1}$ Toshikazu Yamoto ${ }^{1}$ Junya Fukai ${ }^{1}$ \\ Naoyuki Nakao ${ }^{1}$ \\ ${ }^{1}$ Department of Neurological Surgery, Wakayama Medical University, \\ Wakayama, Japan \\ 2 Tokyo Women's Medical University, Institute of Advanced Biomedical \\ Address for correspondence Koji Fujita, Department of Neurological \\ Surgery, Wakayama Medical University, 811-1 Kimiidera, Wakayama \\ 641-0012, Japan (e-mail: kfujita@wakayama-med.ac.jp).
} Engineering and Science, Tokyo, Japan

J Neurol Surg Rep 2014;75:e200-e205.

\begin{abstract}
Objectives Radiation-induced aneurysm is a rare complication for head and neck tumors. Only seven cases of an aneurysm after stereotactic radiosurgery and/or stereotactic radiotherapy (SRT) have been described. We report two patients with a ruptured internal carotid artery (ICA) aneurysm presenting with catastrophic epistaxis after repeated SRT for an anterior skull base tumor.

Keywords

- carotid artery aneurysm

- radiation-induced aneurysm

- stereotactic radiotherapy

- stereotactic radiosurgery

- anterior skull base tumor

Results Two male patients received repeated SRT in various combinations following surgery for an anterior skull base tumor. They presented with significant epistaxis due to rupture of the aneurysm of the ICA 6 and 77 months after the final SRT, respectively. The aneurysms were located within the radiation field. Preoperative angiography had revealed no aneurysms. Thus the aneurysms in these cases were most likely induced by the repeated SRT.

Conclusions This is a proven report of aneurysm formation following repeated SRT without conventional radiotherapy. SRT may be very effective to control malignant skull base tumors. However, the possible development of radiation-induced aneurysm of the ICA should be considered in the case of repeated SRT. The surviving patients who have received SRT should undergo sequential follow-up for possible vascular involvement.
\end{abstract}

\section{Introduction}

Radiation-induced aneurysm is a rare complication for head and neck cancer, and the pathogenesis is not fully understood. ${ }^{1-8}$ But the mechanism is likely to be similar to that of radiation-induced chronic vascular damage. ${ }^{9-12}$

Several authors have reported $\sim 30$ cases of radiationinduced intracranial aneurysms with subsequent rup-

received

July 10,2014

accepted after revision

May 7, 2014

published online

August 5, 2014
DOI http://dx.doi.org/

10.1055/s-0034-1383859. ISSN 2193-6358. ture. ${ }^{1,2,5,8,13}$ These aneurysms were diagnosed from 10 months to 21 years after radiation therapy. So far, only seven cases of aneurysm after stereotactic radiosurgery (SRS) and/or stereotactic radiotherapy (SRT) have been described. ${ }^{14-20}$ All of these patients underwent repeated radiotherapies in various combinations.

We present two cases of catastrophic epistaxis due to a ruptured internal carotid artery (ICA) aneurysm induced by
License terms

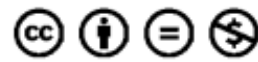

(c) 2014 Georg Thieme Verlag KG
Stuttgart · New York 
repeated SRT for an anterior skull base tumor and successfully treated by endovascular surgery. This is a proven report of aneurysm formation following repeated SRT without conventional radiotherapy. SRT for skull base tumors may be very effective to control these tumors. However, these cases suggest that the development of a radiation-induced aneurysm should be considered a possible serious complication in the case of repeated SRT. Case 1 described here was reported previously in this journal. ${ }^{21}$

\section{Case Reports}

\section{Case 1}

A 29-year-old man was referred to our institution for the treatment of a large Ewing primitive neuroectodermal tumor (PNET) that occupied the right orbit and ethmoid and maxillary sinus with right progressive visual loss in four years earlier ( $\boldsymbol{- F i g . ~ 1 A )}$. The tumor was resected totally via the transbasal approach. Preoperative angiography revealed no abnormality. The right ICA was not injured during the surgery. Postoperatively, the patient underwent adjuvant chemotherapy and SRT with a dose ranging from 45 to 54.9 Gy in 15 fractions that produced a complete response. The right petrous ICA and surrounding structure were located within the radiation field. The recurrent tumor in the right maxillary sinus was seen 28 months after surgery. Therefore, the second
SRT was performed with a dose ranging from 53.5 to $62.3 \mathrm{~Gy}$ in 15 fractions that resulted in the partial response. However, 42 months after surgery, tumor relapse was revealed in the right orbit that remarkably had eroded the sphenoid bone. He underwent the third SRT with a dose ranging from 52.8 to $60.0 \mathrm{~Gy}$ in 15 fractions. Magnetic resonance imaging (MRI) demonstrated the tumor had almost disappeared after SRT (-Fig. 1B), and he was followed regularly as an outpatient.

The patient was admitted to our hospital with complaints of significant epistaxis with hypovolemic shock 6 months after the final SRT. A computed tomography scan revealed a hematoma at the middle cranial fossa and paranasal sinuses, and pneumocephalus (-Fig. 1C). Cerebral angiography showed a right petrous ICA aneurysm with slight stenosis of the adjacent ICA ( - Fig. 1D). Because of rebleeding during the cerebral angiography, we urgently performed endovascular parent-artery occlusion without balloon test occlusion (BTO) to save his life. Cerebral angiography after the endovascular treatment showed complete disappearance of the aneurysm with a tolerable cross-flow via the anterior communicating artery $(\mathrm{ACOA})$ (-Fig. 1E). The postoperative course was uneventful. Postoperative MRI revealed no ischemic or hemorrhagic complications. He recovered fully and was discharged 3 weeks after surgery without new neurologic deficits. But 6 months after endovascular treatment, he died from an untreatable tumor relapse.

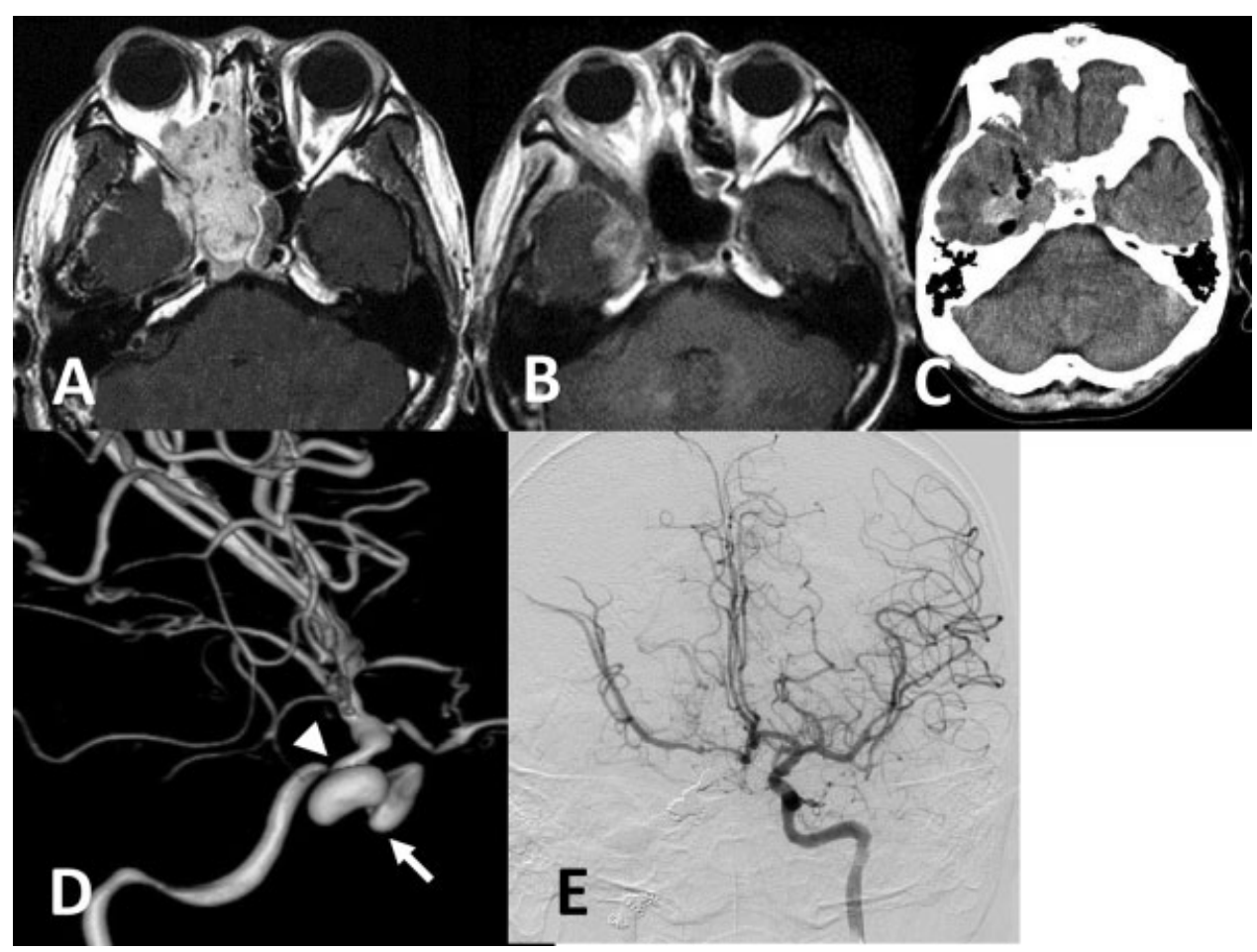

Fig. 1 (A) Axial T1-weighted magnetic resonance image with gadolinium (MRI-Gd) before the surgery revealed the tumor in the orbit and ethmoid sinus adjacent to the right internal carotid artery (ICA). (B) Axial MRI-Gd after the third stereotactic radiotherapy demonstrated remarkable shrinkage of the tumor. (C) Computed tomography scan on emergent admission due to massive epistaxis revealed the hematoma at the middle cranial fossa and pneumocephalus. (D) Right internal carotid angiogram showed a right petrous ICA aneurysm (arrow) with slight stenosis of the adjacent ICA (arrowhead). (E) Left internal carotid angiogram after the endovascular treatment revealed the tolerable cross-flow via the anterior communicating artery. 


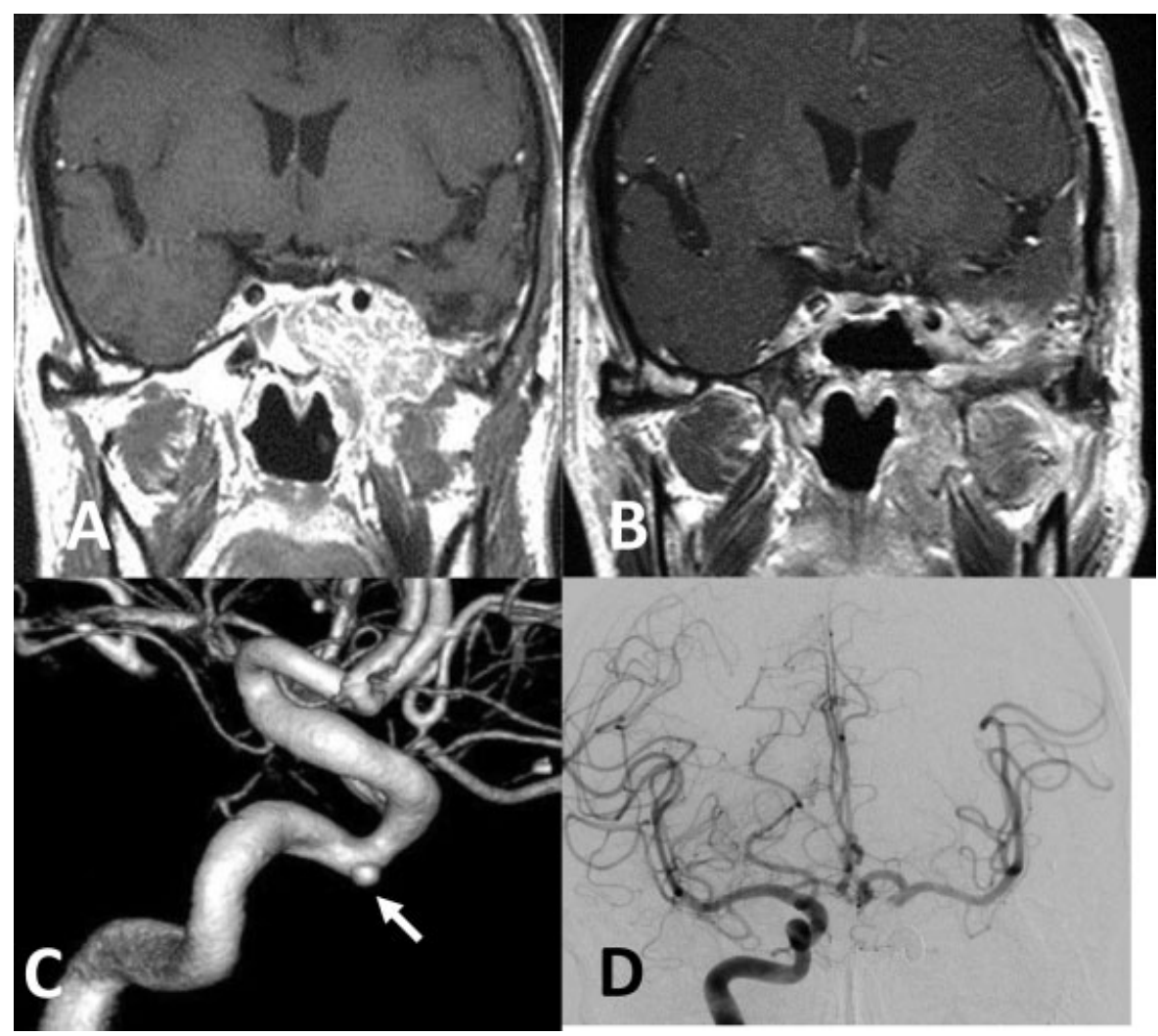

Fig. 2 (A) Coronal T1-weighted magnetic resonance image with gadolinium (MRI-Gd) after stereotactic radiosurgery revealed tumor recurrence in the cavernous sinus and the left infratemporal fossa surrounding the left internal carotid artery (ICA). (B) MRI-Gd after stereotactic radiosurgery and stereotactic radiotherapy demonstrated an almost disappearance of the tumor. (C) Left internal carotid angiogram demonstrated a small aneurysm at the left cavernous ICA (arrow). (D) Right internal carotid angiogram after the endovascular treatment showed enough collateral flow via the anterior communicating artery.

\section{Case 2}

A 61-year-old man was admitted to our hospital for the treatment of a left large sphenocavernous meningioma in 1999 at 48 years of age. The tumor was partially resected via the orbitozygomatic approach without removal of the cavernous mass. The left ICA was not damaged during the surgery. The pathologic diagnosis was atypical meningioma. The left cavernous tumor had gradually grown 23 months after the initial surgery and was treated by SRS using gamma knife. The tumor margin was covered by the $50 \%$ isodose line, and 12 Gy were delivered to the margin. The left cavernous ICA was located within the radiation field. However, the rest of the tumor continued to grow to the infratemporal fossa (-Fig. 2A). Two surgeries, using the infratemporal fossa approach and transsphenoidal approach, were required to remove the extracranial tumor without removal of the cavernous tumor 83 months after the first operation. Preoperative angiography revealed no abnormality. The left ICA was not injured during the operations. The following month, the patient underwent SRT using cyber knife with a dose ranging from 30.0 to $47.6 \mathrm{~Gy}$ in five fractions that produced a complete response (-Fig. 2B). He was followed up on an outpatient basis.

The patient was admitted to our hospital with complaints of massive epistaxis 77 months after the last SRT. Cerebral angiography showed a small aneurysm located at the cavern- ous portion of the left ICA (- Fig. 2C). BTO of the left ICA demonstrated the presence of collateral flow via the ACoA without a reduced regional cerebral oxygenation state on the occluded side under the monitoring of near-infrared spectroscopy. Therefore, we decided to perform endovascular internal trapping of the left ICA. Cerebral angiography after the endovascular treatment showed complete disappearance of the aneurysm with enough collateral flow via the ACoA (-Fig. 2D). The postoperative course was uneventful. Postoperative MRI revealed no complications. He was discharged 2 weeks after the endovascular treatment without any new neurologic deficits.

\section{Discussion}

Radiation-induced carotid artery aneurysm is a rare complication of radiation therapy for head and neck carcinoma, but it is a life-threatening situation. The pathogenesis of radiation-induced aneurysm remains speculative. ${ }^{1-8}$ However the mechanism is likely to be similar to that of radiation-induced vasculopathy, due to chronic vascular damage such as obstruction of the vasa vasorum, premature atherosclerosis, adventitial fibrosis, and necrosis of the arterial wall. ${ }^{9-12}$ Combined with high blood pressure of the great vessel, it could result in the rupture of the arterial wall and even dissection with extravasation blood. ${ }^{13,22,23}$ Therefore, even 
Ruptured ICA Aneurysm after Repeated SRT Fujita et al. e203

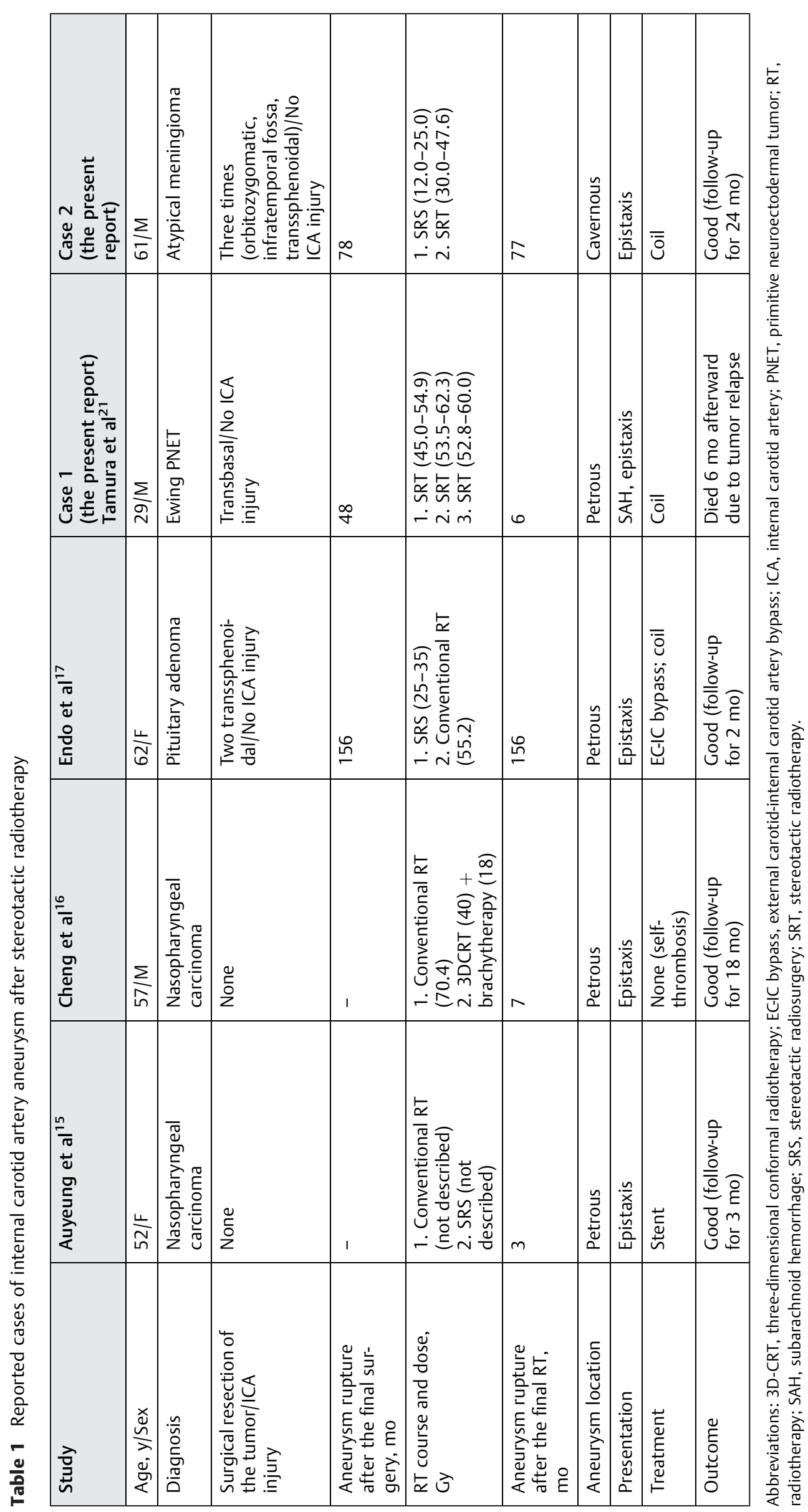


a small radiation-induced aneurysm is more prone to rupture than other common saccular aneurysms. ${ }^{24}$

The clinical characteristics of radiation-induced aneurysms differ from other saccular aneurysms. ${ }^{13}$ Radiationinduced aneurysms originate directly from the arterial wall, which is less likely to be influenced by the direction of the blood flow, in contrast to other saccular aneurysms. Therefore, the formation of an aneurysm after irradiation might occur at any location on the vessel included within the radiation fields. In our cases, these aneurysms were not located at a branching site and were included within the radiation field. The angiography before the initial surgery revealed no aneurysm formation. In addition, the carotid arteries were not injured during the surgery. These findings suggest that the SRT was responsible for the formation of the carotid artery aneurysm.

Several authors have reported $\sim 30$ cases of radiationinduced intracranial aneurysms with subsequent rupture. ${ }^{1,2,5,8,13}$ These aneurysms were diagnosed from 10 months to 21 years after radiation therapy. However, our literature review yielded only seven cases of aneurysm induced by SRS and/or SRT. ${ }^{14-20}$

Among them, four cases were presented as anterior inferior cerebellar artery aneurysm induced by SRS (gamma knife) for vestibular schwannoma. ${ }^{14,18-20}$ The remaining three cases were reported as ICA aneurysms ${ }^{15-17}$ (-Table1). With the inclusion of our cases, these patients were three men and two women (mean age: 52.2 years; range: 29-62 years). There were two cases of nasopharyngeal carcinoma, and one each of pituitary adenoma, atypical meningioma, and Ewing PNET. Three patients underwent operations without ICA injury before the final radiotherapy. All patients received repeated radiotherapies in various combinations. In addition, only our cases received repeated SRS or SRT without conventional radiotherapy. Although the interval between the latest radiotherapy and the diagnosis of the aneurysm varied from 3 months to 13 years (mean: 49.8 months), three cases developed within a year. These results indicated that repeated SRT causes an aneurysm to occur earlier than conventional radiotherapy or single SRT.

SRT makes it possible to deliver efficacious therapeutic doses accurately to the targeted lesion with secure preservation of adjacent normal anatomical structures. However, if these structures are located within the targeted lesion, they must be involved in the radiation field. Excessive doses into them by repeated SRT can lead to critical complications. Therefore the surviving patients who have received SRT should undergo sequential follow-up for possible vascular involvement.

Treatment for radiation-induced aneurysms includes clipping, ${ }^{2,3,5,24-26}$ trapping, ${ }^{11,27}$ and wrapping the aneurysm and parent artery. ${ }^{2,5,28,29}$ In addition, parent artery occlusion using an endovascular technique is effective. ${ }^{19,28,30}$ Because of the high risk of mortality, if the radiation-induced aneurysm ruptures, aggressive surgical intervention or endovascular treatment should be considered for the aneurysm. Sacrifice of the carotid artery would lead to severe cerebrovascular events, and therefore a BTO should be performed. If a
BTO is successful, trapping or parent artery occlusion becomes an option. If the patient cannot tolerate the test, an extracranial-to-intracranial bypass surgery should be considered. In case 1 of our series, the patient was semicomatose and in a state of hypovolemic shock due to repeated massive epistaxis. Therefore, an immediate salvage procedure was needed to save his life without performing BTO. In case 2 , the patient could tolerate the test adequately. Hence ICA was sacrificed for parent artery occlusion.

We have presented two cases of a ruptured ICA aneurysm induced by repeated SRT for anterior skull base tumor. It was definitely difficult to clarify accurately the timing of aneurysm formation in our cases. In addition, although the primary factor was presumed the high dose delivered to the ICA by the repeated SRT, an unrecognized carotid injury at the former surgical intervention to the tumor or direct damage to the vessel wall by the tumor could also have been the probable causes. There are some reports on iatrogenic intracranial traumatic aneurysms after the resection of skull base tumors. ${ }^{31-33}$ It was suggested that most traumatic intracranial aneurysms have a symptomatic onset within 4 weeks after injury of the vessel wall. ${ }^{32}$ In our cases, however, the hemorrhagic presentation appeared 48 and 78 months after the final surgery, respectively. Therefore, we are convinced that the aneurysms in our cases were most likely induced by the repeated SRT and indicated a low probability of traumatic origin. Histologic analysis, if possible, might contribute to an understanding of the entity of these lesions.

\section{Conclusions}

We present two cases of catastrophic epistaxis due to ICA aneurysm that were most likely induced by repeated SRT for anterior skull base tumors. The SRT may be very effective to control the residual tumor as well as tumor relapse. However, the possible development of radiation-induced aneurysm of the carotid artery should be considered in the case of repeated SRT for malignant skull base tumors.

\section{References}

1 Azzarelli B, Moore J, Gilmor R, Muller J, Edwards M, Mealey J. Multiple fusiform intracranial aneurysms following curative radiation therapy for suprasellar germinoma. Case report. J Neurosurg 1984;61(6):1141-1145

2 Casey AT, Marsh HT, Uttley D. Intracranial aneurysm formation following radiotherapy. Br J Neurosurg 1993;7(5): 575-579

3 Jensen FK, Wagner A. Intracranial aneurysm following radiation therapy for medulloblastoma. A case report and review of the literature. Acta Radiol 1997;38(1):37-42

4 Lee JK, Chelvarajah R, King A, David KM. Rare presentations of delayed radiation injury: a lobar hematoma and a cystic spaceoccupying lesion appearing more than 15 years after cranial radiotherapy: report of two cases. Neurosurgery 2004;54(4): 1010-1013; discussion 1013-1014

5 Maruyama K, Mishima K, Saito N, Fujimaki T, Sasaki T, Kirino T. Radiation-induced aneurysm and moyamoya vessels presenting with subarachnoid haemorrhage. Acta Neurochir (Wien) 2000; 142(2):139-143 
6 Murros KE, Toole JF. The effect of radiation on carotid arteries. A review article. Arch Neurol 1989;46(4):449-455

7 O'Connor MM, Mayberg MR. Effects of radiation on cerebral vasculature: a review. Neurosurgery 2000;46(1):138-149; discussion $150-151$

8 Pereira P, Cerejo A, Cruz J, Vaz R. Intracranial aneurysm and vasculopathy after surgery and radiation therapy for craniopharyngioma: case report. Neurosurgery 2002;50(4):885-887; discussion $887-888$

9 Kiyosue H, Okahara M, Tanoue S, et al. Dispersion of coils after parent-artery occlusion of radiation-induced internal carotid artery pseudoaneurysm. AJNR Am J Neuroradiol 2004;25(6):1080-1082

10 Okamura HO, Kamiyama R, Takiguchi Y, Kimizuka K, Ishikawa N, Kishimoto S. Histopathological examination of ruptured carotid artery after irradiation. ORL J Otorhinolaryngol Relat Spec 2002; 64(3):226-228

11 Sciubba DM, Gallia GL, Recinos P, Garonzik IM, Clatterbuck RE. Intracranial aneurysm following radiation therapy during childhood for a brain tumor. Case report and review of the literature. J Neurosurg 2006;105(2, Suppl):134-139

12 Silverberg GD, Britt RH, Goffinet DR. Radiation-induced carotid artery disease. Cancer 1978;41(1):130-137

13 Benson PJ, Sung JH. Cerebral aneurysms following radiotherapy for medulloblastoma. J Neurosurg 1989;70(4):545-550

14 Akamatsu Y, Sugawara T, Mikawa S, et al. Ruptured pseudoaneurysm following gamma knife surgery for a vestibular schwannoma. J Neurosurg 2009;110(3):543-546

15 Auyeung KM, Lui WM, Chow LC, Chan FL. Massive epistaxis related to petrous carotid artery pseudoaneurysm after radiation therapy: emergency treatment with covered stent in two cases. AJNR Am J Neuroradiol 2003;24(7):1449-1452

16 Cheng KY, Lee KW, Chiang FY, Ho KY, Kuo WR. Rupture of radiation-induced internal carotid artery pseudoaneurysm in a patient with nasopharyngeal carcinoma-spontaneous occlusion of carotid artery due to long-term embolizing performance. Head Neck 2008;30(8):1132-1135

17 Endo $\mathrm{H}$, Fujimura M, Inoue $\mathrm{T}$, et al. Simultaneous occurrence of subarachnoid hemorrhage and epistaxis due to ruptured petrous internal carotid artery aneurysm: association with transsphenoidal surgery and radiation therapy: case report. Neurol Med Chir (Tokyo) 2011;51(3):226-229

18 Park KY, Ahn JY, Lee JW, Chang JH, Huh SK. De novo intracranial aneurysm formation after gamma knife radiosurgery for vestibular schwannoma. J Neurosurg 2009;110(3):540-542

19 Takao T, Fukuda M, Kawaguchi T, et al. Ruptured intracranial aneurysm following gamma knife surgery for acoustic neuroma. Acta Neurochir (Wien) 2006;148(12):1317-1318; discussion 1318

20 Yamaguchi S, Kato T, Takeda M, Ikeda H, Kitamura K. Ruptured distal anterior inferior cerebellar artery aneurysm following stereotactic irradiation for vestibular schwannoma: case report. Neurol Med Chir (Tokyo) 2009;49(5):202-205
21 Tamura M, Kogo K, Masuo O, et al. Formation and rupture of the internal carotid artery aneurysm after multiple courses of intensity-modulated radiation therapy for management of the skull base Ewing sarcoma/PNET: Case report. J Neurol Surg Rep 2013; 74(2):111-117

22 Chen HC, Lin CJ, Jen YM, et al. Ruptured internal carotid pseudoaneurysm in a nasopharyngeal carcinoma patient with skull base osteoradionecrosis. Otolaryngol Head Neck Surg 2004;130(3): 388-390

23 Mok JS, Marshall JN, Chan M, van Hasselt CA. Percutaneous embolization to control intractable epistaxis in nasopharyngeal carcinoma. Head Neck 1999;21(3):211-216

24 Gonzales-Portillo GA, Valdivia JM. Uncommon presentation of pediatric ruptured intracranial aneurysm after radiotherapy for retinoblastoma. Case report. Surg Neurol 2006;65(4):391-395; discussion 395-396

25 Aichholzer M, Gruber A, Haberler C, Bertalanffy A, Slavc I, Czech T. Intracranial hemorrhage from an aneurysm encased in a pilocytic astrocytoma-case report and review of the literature. Childs Nerv Syst 2001;17(3):173-178

26 Yucesoy K, Feiz-Erfan I, Spetzler RF, Han PP, Coons S. Anterior communicating artery aneurysm following radiation therapy for optic glioma: report of a case and review of the literature. Skull Base 2004;14(3):169-173

27 McConachie NS, Jacobson I. Bilateral aneurysms of the cavernous internal carotid arteries following yttrium-90 implantation. Neuroradiology 1994;36(8):611-613

28 Murakami N, Tsukahara T, Toda H, Kawakami O, Hatano T. Radiation-induced cerebral aneurysm successfully treated with endovascular coil embolization. Acta Neurochir Suppl (Wien) 2002; 82:55-58

29 Nishi T, Matsukado Y, Kodama T, Hiraki T. Multiple intracranial aneurysms following radiation therapy for pituitary adenoma. Case report. [in Japanese]. Neurol Med Chir (Tokyo) 1987;27(3): 224-228

30 Cheng KM, Chan CM, Cheung YL, Chiu HM, Tang KW, Law CK. Endovascular treatment of radiation-induced petrous internal carotid artery aneurysm presenting with acute haemorrhage. A report of two cases. Acta Neurochir (Wien) 2001;143(4):351-355; discussion 355-356

31 Ciceri EFM, Regna-Gladin C, Erbetta A, et al. latrogenic intracranial pseudoaneurysms: neuroradiological and therapeutical considerations, including endovascular options. Neurol Sci 2006;27(5): 317-322

32 Mathews SS, Kumar RM, Rupa V. Iatrogenic pseudoaneurysm: a rare complication of sinonasal surgery. Am J Otolaryngol 2011; 32(6):607-610

33 Chen Z, Zhang J, Miao H, Niu Y, Feng H, Zhu G. Delayed rupture of iatrogenic cerebral pseudoaneurysms after neurosurgical procedures: report of two cases. Clin Neurol Neurosurg 2013;115(8): 1552-1554 